\title{
A New Linear Parametrization for Peak Friction Coefficient Estimation in Real Time
}

\author{
Ricardo de Castro, Rui Esteves Araujo, Jaime S. Cardoso, Diamantino Freitas \\ Faculty of Engineering, University of Porto \\ R. Dr. Roberto Frias, 4200-465 Porto, Portugal \\ \{de.castro, raraujo, jsc, dfreitas\}@fe.up.pt
}

\begin{abstract}
The correct estimation of the friction coefficient in automotive applications is of paramount importance in the design of effective vehicle safety systems. In this article a new parametrization for estimating the peak friction coefficient, in the tire-road interface, is presented. The proposed parametrization is based on a feedforward neural network (FFNN), trained by the Extreme Learning Machine (ELM) method. Unlike traditional learning techniques for FFNN, typically based on backpropagation and inappropriate for real time implementation, the ELM provides a learning process based on random assignment in the weights between input and the hidden layer. With this approach, the network training becomes much faster, and the unknown parameters can be identified through simple and robust regression methods, such as the Recursive Least Squares. Simulation results, obtained with the CarSim program, demonstrate a good performance of the proposed parametrization; compared with previous methods described in the literature, the proposed method reduces the estimation errors using a model with a lower number of parameters.
\end{abstract}

\section{INTRODUCTION}

In automotive applications, the adhesion conditions present in the tire-road interface, typically characterized by the coefficient of friction, have a strong influence in the vehicle behavior and safety. In recent years, with the proliferation of active safety systems (like ABS, TCS, ESP and VDC) [1], the estimation of the friction coefficient has attracted a growing interest in the research community, since the knowledge of this variable contributes significantly to increasing the effectiveness of the vehicle safety systems. Additionally, intelligent vehicles, as is the case of autonomous vehicles, currently at an early stage of development, can also benefit from the friction estimation, adapting the control strategies to the maximum grip levels available on the road [2].

Unlike other easily measurable variables, such as the vehicle acceleration, yaw rate and wheel speeds, currently, there is no economically viable sensor that could be fitted in the vehicle to measure the friction coefficient. These difficulties have encouraged the development of virtual sensors to estimate this variable using easily measurable signals. In addition to the immeasurable factor, the real-time requirements associated with this application poses strong constrains on the model complexity. Non-linear parameterizations, such as the Magic Tire Formula [3] or the Burckhardt model [4] should be avoided because of the difficulty in identifying non-linear models in real-time. Therefore, linear parameterizations, like

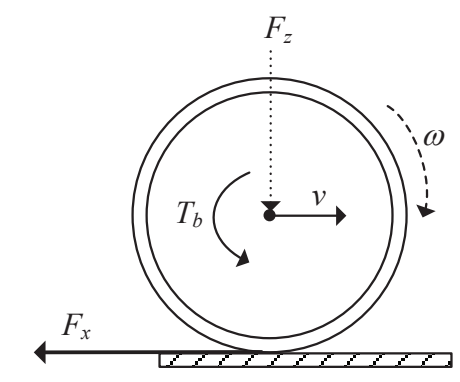

Figure 1. Simplified quarter car model.

polynomials [5], [6] or fixed exponentials [7] should be privileged for real-time implementations.

Motivated by the good approximation properties offered by Neural Networks (NN) [8], in this article we explore the possibility of approximate the friction coefficient function $\mu($.$) using a NN model. One of the major disadvantages$ in this type of models is the high processing power required by conventional learning techniques, typically based on back-propagation and clearly inappropriate for real time implementation. To overcome this difficulty, we applied the Extreme Learning Machine (ELM) method [9], [10], which allowed us to transform the non-linear NN model in a linear parametrization, with a reduced complexity, easier and faster to train. Compared with other linear parameterizations presented in the literature [4]-[7], the method proposed in this article is shown to offer better estimation performance with less number of parameters, thus easier to identify in real-time.

\section{Review of Model And Estimation Methods}

A brief introduction to the friction estimation methodology is provided in this section. We start with a description of a simplified vehicle model and then present the main methodologies for estimating de friction coefficient in the tire-road interface, focusing on static based methods.

\section{A. Simplified Vehicle Model}

The dynamic behavior of the vehicle was modeled by the simplified quarter car model (see Figure 1), widely used in the literature of the area [4], [7], [11]:

$$
\begin{aligned}
J \dot{\omega} & =r F_{x}-T_{b} \\
M \dot{v} & =-F_{x}
\end{aligned}
$$


where $\omega$ represents the wheel angular speed, $v$ is the longitudinal vehicle speed, $T_{b}$ is the braking torque applied to the wheel, $F_{x}$ is the friction force between tire and the road, $J$ is the wheel and transmission inertia, $M$ is the equivalent mass coupled to the wheel and $r$ is the wheel radius. For simplicity, in this work we only consider the braking maneuvers, with $v>r \omega$, but the obtained results can be easily modified for acceleration maneuvers.

Modeling the friction force $F_{x}$ is the main difficulty in the relation (1). Generally, the friction force is proportional to the normal force that the wheel supports $\left(F_{z}\right)$ and depends on a nonlinear function $\mu($.$) , known as the friction coefficient,$ which varies with the longitudinal tire slip $(\lambda)$, road adhesion conditions, tire pressure, temperature, wear, among other factors, and can be grouped in a parameter vector $\boldsymbol{\beta} \in \mathbb{R}^{d}$ :

$$
\begin{aligned}
F_{x} & =F_{z} \mu(\lambda, \boldsymbol{\beta}) \\
\lambda & =\frac{v-\omega r}{v}
\end{aligned}
$$

The most popular approaches to represent the friction coefficient $\mu($.$) are based on two types of models: (i) static and (ii)$ dynamic. The static models, like the Burckhardt [4] model:

$$
\mu(\lambda, \boldsymbol{\beta})=\beta_{1}\left(1-e^{-\beta_{2} \lambda}\right)-\beta_{3} \lambda
$$

and the magic tyre formula (MTF) [3]:

$\mu(\lambda, \boldsymbol{\beta})=\beta_{1} \sin \left(\beta_{2} \operatorname{atan}\left(\left(1-\beta_{4}\right) \beta_{3} \lambda+\beta_{4} \operatorname{atan}\left(\beta_{3} \lambda\right)\right)\right)$

where the vector $\boldsymbol{\beta}=\left[\begin{array}{llll}\beta_{1} & \beta_{2} & \ldots & \beta_{d}\end{array}\right]^{T}$ represents the model parameters, were developed by applying curve fitting techniques to the experimental tire data. The great advantage of the static models is their simplicity, but, on the other hand, they are limited to steady state conditions and the model parameters lack physical meaning. More recently, dynamic models, like the LuGre [11], were applied to represent the tire/road friction, presenting promising features to capture the transient behavior of the friction. A complete review of the friction models is beyond the scope of this work, and a detailed discussion about this topic can be found on [12] (and references therein). In this work we focused on the static models to represent the friction coefficient.

Throughout the document we refer to $\mu(\lambda, \boldsymbol{\beta})$ as non-linear parameterizations, like (4) and (5). Linear approximations are defined as $\hat{\mu}(\lambda, \boldsymbol{\theta})$, which are linear in the $\boldsymbol{\theta} \in \mathbb{R}^{m}$ and are used to approximate the non-linear parametrization in a subset $\lambda \in \mathcal{K} \subset[0,1], \boldsymbol{\beta} \in \mathcal{D} \subset \mathbb{R}^{d}$.

\section{B. Qualitative vs Quantitative Estimation}

The estimating process of the friction coefficient, based on static models, can be divided in two categories: qualitative and quantitative. In both cases, the main objective is to obtain an estimation of the peak friction coefficient, but the output of the two mentioned methods is very different. In the first case, qualitative, the output of the estimator is based on a grading system, providing an indicator of adhesion quality, for instance qualifying the grip levels in a grading scale from 1 (very

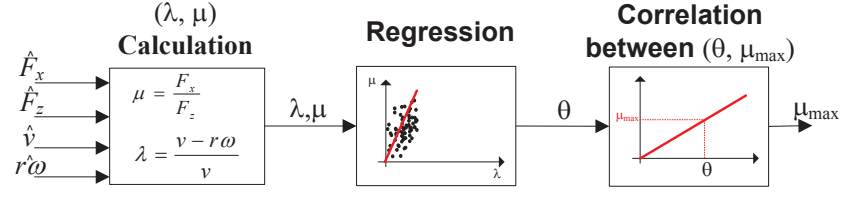

Figure 2. Block diagram of qualitative peak friction estimator, based on static models.

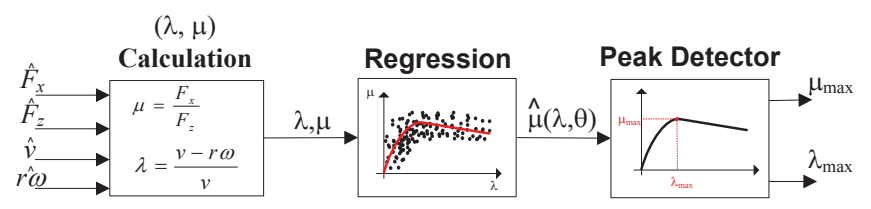

Figure 3. Block diagram of quantitative peak friction estimator, based on static models.

slippery) to 5 (high grip); in the second case, quantitative, a numeric output is generated to quantify the adhesion.

Examples of the qualitative approach can be found on references [13]-[16]. The main driving force behind these approaches is the problem of persistence of excitation: in order to extract the peak friction we need to apply high levels of tire slip, which is not desirable from the safety point of view. To avoid this problem, the qualitative methods identify the tyre longitudinal stiffness $\left(\approx \partial \mu /\left.\partial \lambda\right|_{\lambda \rightarrow 0}\right)$, using samples with low slip values, and, then, try to correlate them with the peak friction (see Figure 2). Albeit these approaches solve the problem of persistence of excitation, they also introduce a new issue: correlating the longitudinal stiffness with the peak friction. This correlation is very hard to obtain in practice and, as pointed out by [13] and [17], varies with the type of tires, tire wear, pressure and temperature, among many other factors. Therefore, it is still very difficult to apply these qualitative approaches in practice.

On the other hand, the quantitative methods [4]-[7], detailed in Section II-D, offer a much simpler algorithm to extract the peak friction. Although the estimation is obtained at expense of applying high tire slips, the estimation process is more robust and also offer the possibility to identify the optimal slip reference $\lambda_{\max }$, which is very useful for some anti-lock braking and traction control systems [7][18].

In the authors' opinion, both methodologies have merits and drawbacks which are difficult to balance, and it is not yet clear which approach will prevail. In this article we offer a contribution to the quantitative methods, by introducing a new parametrization with less number of parameters, providing some practical advantages.

\section{Estimation methodology}

It is interesting to note that, although the output of the qualitative and quantitative estimation methods are different, they have similar structures that can be systematized in three steps (see Figure 2 and 3). In first place we need to collect experimental samples of tire slip and friction coefficient; 
then a parametric representation must be estimated to fit the experimental data and finally the maximum friction is extracted.

1) Collect Samples: the tire slip $(\lambda)$ and the instantaneous friction coefficient $\left(\mu=F_{x} / F_{z}\right)$ are variables very difficult to measure experimentally. For instance, $\lambda$ depend on the vehicle speed and $\mu$ on the longitudinal force, which are known to be very expensive to measure directly. In this work we assume that these variables are available to the estimation process, for example using vehicle speed observers [4] and estimating the tire longitudinal force with relations easily derived from (2), like $\hat{F}_{x}=\left(J \dot{\omega}+T_{b}\right) / r$. These experimental samples can be grouped in a set of $N$ input/output samples: $\left\{\left(\lambda_{i}, \mu_{i}\right)\right\}_{i=1 . . N}$.

2) Regression Method: after collecting the experimental samples, a parametric representation for the static friction coefficient must be obtained. Normally, linear parameterizations $\hat{\mu}(\lambda, \boldsymbol{\theta})$ are favored because they are simpler and faster to identify. The parameters of the model can be found applying regression techniques:

$$
\psi:\left\{\left(\lambda_{i}, \mu_{i}\right)\right\}_{i=1 . . N} \longmapsto \boldsymbol{\theta}
$$

where $\psi$ represents the regression method (such as recursive least squares [4], total least squares [17], maximum likelihood [7], etc.) used to obtain the model parameters $\boldsymbol{\theta}$. Normally, the parameterizations used in the qualitative estimation have less number of free parameters than the quantitative methods. For example, in a qualitative method the tire longitudinal stiffness can be detected with a 2 parameters model (a gain and offset) [13], while in the quantitative a more complex model must be used to approximate the full non-linear friction curve.

3) Peak friction detection: finally, after calculating the model parameters, $\boldsymbol{\theta}$, the peak friction coefficient must be extracted. In the quantitative case, this is easily performed computing the function maximum:

$$
\begin{aligned}
\hat{\mu}_{\max } & =\max _{\lambda} \hat{\mu}(\lambda, \boldsymbol{\theta}) \\
\hat{\lambda}_{\max } & =\underset{\lambda}{\arg \max } \hat{\mu}(\lambda, \boldsymbol{\theta})
\end{aligned}
$$

while in the qualitative case a correlation between the parameters and a adhesion grading system must be applied, for instance: $\hat{\mu}_{\max }: \boldsymbol{\theta} \rightarrow\{$ very slippery, ..., high grip $\}$. From the implementation perspective, the main difference between the qualitative and quantitative methods is in the final step.

\section{Previous Linear Parameterizations}

In this section we describe previous efforts, presented in the literature, to linear approximate the static friction coefficient function in the quantitative methods. Due to the simplicity of the Burckhardt model, most of the approximation methods found on the literature try to get a linear parametrization for (4). One of the first practical solutions to this problem was proposed by Kiencke [5]:

$$
\hat{\mu}(\lambda, \boldsymbol{\theta})=\frac{\lambda}{\theta_{1}+\theta_{2} \lambda+\theta_{3} \lambda^{2}}
$$

Although the parametrization is non-linear, it should noted that by multiplying both sides of the equation with $\theta_{1}+\theta_{2} \lambda+\theta_{3} \lambda^{2}$, the model become linear on the parameters $\theta_{i}, i=1,2,3$ [2]; thus it can be cataloged as a linear identifiable model. Another similar approach, proposed by Germann [6], uses a second order polynomial to approximate the curve $\mu($.$) for slips$ inferior to 0.3 :

$$
\hat{\mu}(\lambda, \boldsymbol{\theta})=\theta_{1}+\theta_{2} \lambda+\theta_{3} \lambda^{2}
$$

The main motivation behind the introduction of these models was to estimate the $\mu_{\max }$ value, a task in which they provide good performance. However, these parameterizations have some difficulties when estimating the $\lambda_{\max }$ variable, which is very important for generating the optimal slip reference for anti-lock braking and traction control systems [18]. To overcome this difficulty, recently, Tanelli et al. [7] proposed two alternatives. In the first one, the non-linear Burckhard model is kept, but the model parameters are estimated using the maximum likelihood approach. Although this approach provides satisfactory estimation errors, the convergence of the estimation algorithm is difficult to guarantee, which motivated the introduction of a new linear model to approximate (4). The parametric space under consideration was characterized by:

$$
\boldsymbol{\beta}=\left[\begin{array}{lll}
\beta_{1} & \beta_{2} & \beta_{3}
\end{array}\right]^{T} \in \mathbb{R} \times[4,100] \times \mathbb{R}
$$

corresponding to the main types of conditions found in practice. Tanelli et al. [7] noticed that the main non-linearity in (4) is the exponential function $e^{-\beta_{2} \lambda}$, with $\beta_{2} \in[4,100]$, and proposed a simple approximation using fixed exponentials, equally spread in the interval $[4,100]$. The resulting linear parametrization is defined as:

$$
\hat{\mu}(\lambda, \boldsymbol{\theta})=\left[\begin{array}{llllll}
1 & \lambda & e^{-4 \lambda} & e^{-36 \lambda} & e^{-68 \lambda} & e^{-100 \lambda}
\end{array}\right] \boldsymbol{\theta}
$$

which is characterized by 6 basis functions. Despite the promising results obtained with this model, we can ask if it is possible to develop other linear parameterizations using fewer basis functions and providing equivalent performance. The number of basis functions, which is also equal to the number of unknown parameters, has an important practical effect: models with less number of unknown parameters tend to be easier to tune and estimate. Stimulated by this issue, in the next section we investigate the ELM approach as a method to develop new linear parametrization for the static friction coefficient.

\section{THE ELM BASED PARAMETRIZATION}

It is a well known fact that feedforward neural networks (FFNN), with a single hidden-layer, are capable of approximate complex nonlinear functions using input/output samples, and represent universal function approximators [8]. These networks, with scalar inputs and outputs, can be described as:

$$
\begin{aligned}
\hat{\mu}(\lambda, \boldsymbol{\theta}) & =\sum_{i=1}^{N_{c}} \theta_{i} h_{i}\left(\lambda, w_{i}, b_{i}\right) \\
h_{i}\left(\lambda, w_{i}, b_{i}\right) & =g\left(w_{i} \lambda+b_{i}\right)
\end{aligned}
$$


where $N_{c}$ represent the number of nodes in the network and the basis functions $h_{i}$ depends on the weights $w_{i} \in \mathbb{R}$, bias $b_{i} \in \mathbb{R}$ and the activation function in the hidden layer $g: \mathbb{R} \rightarrow \mathbb{R}$ (sigmoid, linear, etc.). In (11) the output activation function is assumed linear, as standard practice for regression models. Traditionally, during the training phase, all parameters in the network, i.e. $w_{i}, b_{i}$ and $\theta_{i}$, must be simultaneously adapted using gradient based searches. However, this approach requires a high computational power, with consequent increase in processing times and unsuitable for real time implementation.

To attenuate these drawbacks, Huang [9], [10] proposed a new learning strategy, designated as Extreme Learning Machine (ELM), suitable for fast training of a FFNN with a single hidden-layer. The ELM simplifies the training phase by randomly assigning the weights $w_{i}$ and bias $b_{i}$, producing linear models in the $\theta_{i}$ parameters, which are easily trained using robust regression techniques, like least squares. It can be shown that if: $i$ ) the FFNN has a number nodes $N_{c}$ equal to the number of input/output samples $N$; ii) randomly assigned weights $w_{i}$ and bias $b_{i}$; and iii) $g$ is infinitely differentiable; then (11) is able to approximate all the training samples with zero error (a formal proof can be found on [9]). In the $\mu$ estimation problem, normally, the input/output samples are corrupted with noise, and the zero error training is not a hard requirement. Therefore, a model reduction can be obtained, selecting a $N_{c}$ inferior to the number of samples $N$. Compared with the traditional backpropagation training method, the ELM can offer a much faster learning speed, with less overfitting and better generalizing performance [9].

Based on the theoretical results offered by the ELM, a FFNN based linear parametrization of the friction coefficient is guaranteed to exist. We have found that, using (11) with sigmoid activation functions, i.e. $g(x)=1 /\left(1+e^{-x}\right)$, and random selection of $w_{i}, b_{i}$ generated by a normal distribution $\left(w_{i} \in \mathcal{N}\left(0,10^{2}\right)\right.$ and $\left.b_{i} \in \mathcal{N}\left(0,1^{2}\right)\right)$, a reasonable approximation of the Burckhardt function can be obtained with only 8 nodes $\left(N_{c}=8\right)$. Nevertheless, the use of completely random values in the parameters $w_{i}, b_{i}$ results in parameterizations with a non-minimum number of nodes. To reduce the number of nodes in the FFNN we selected the most representative curves of the Burckhardt model (dry and wet asphalt, cobbles and snow) [4] and looked for the best parameters $w_{i}, b_{i}$ that can generate lower fitting errors and number of nodes. This compromise was obtained with $N_{c}=4$, and the resulting model is defined as:

$$
\hat{\mu}(\lambda, \boldsymbol{\theta})=\sum_{i=1}^{4} \theta_{i} \frac{1}{1+e^{-\left(w_{i} \lambda+b_{i}\right)}}
$$

with

$$
\begin{aligned}
{\left[\begin{array}{llll}
w_{1} & w_{2} & w_{3} & w_{4}
\end{array}\right] } & =\left[\begin{array}{llll}
-29.78 & -11.78 & 1.41 & 4.94
\end{array}\right] \\
{\left[\begin{array}{lllll}
b_{1} & b_{2} & b_{3} & b_{4}
\end{array}\right] } & =\left[\begin{array}{llll}
-0.89 & 0.49 & 0.07 & 1.65
\end{array}\right]
\end{aligned}
$$

It should be emphasized that, compared with the Tanelli et al. [7] parametrization, the model proposed in this article has

\begin{tabular}{|c|c|c|c|}
\hline Name & Model $\mu(\lambda)$ & $\mathbf{N}^{1}$ & Reg. Method ${ }^{2}$ \\
\hline Kiencke2 [4] & $\frac{\lambda}{K+\theta_{1} \lambda+\theta_{2} \lambda^{2}}, \quad k \simeq 1 / 30$ & 2 & LS, RLS \\
\hline Kiencke3 [4] & $\frac{\lambda}{\theta_{1}+\theta_{2} \lambda+\theta_{3} \lambda^{2}}$ & 3 & LS, RLS \\
\hline Germann [6] & $\theta_{1}+\theta_{2} \lambda+\theta_{3} \lambda^{2}$ & 3 & LS, RLS \\
\hline Tanelli_a [7] & $\theta_{1}\left(1-e^{-\theta_{2} \lambda}\right)-\theta_{3} \lambda$ & 3 & ML \\
\hline Tanelli_b [7] & $\sum_{k=1}^{4} \theta_{k} e^{-b_{k} \lambda}+\theta_{5} \lambda+\theta_{6}$ & 6 & LS, RLS \\
\hline ELM & $\sum_{k=1}^{4} \theta_{k}\left(1+e^{-w_{k} \lambda-b_{k}}\right)^{-1}$ & 4 & LS, RLS \\
\hline
\end{tabular}

Table I

LINEAR PARAMETERIZATIONS EVALUATED IN THIS ARTICLE

Table II

MAGiC TyRe Formula (MTF) PARAMETERS USED TO ARTIFICIALLY GENERATE TEST SAMPLES.

\begin{tabular}{|c|c|c|c|c|}
\hline Condition & $\beta_{1}$ & $\beta_{2}$ & $\beta_{3}$ & $\beta_{4}$ \\
\hline Asph. Dry & 1.0 & 2 & 0.08 & 0.90 \\
Asph. Wet & 0.6 & 2 & 0.10 & 0.90 \\
Cobbles & 0.8 & 2 & 0.04 & 1.00 \\
Snow & 0.2 & 2 & 0.15 & 0.95 \\
\hline
\end{tabular}

a reduced number of parameters (4), with practical benefits, as previously discussed. For estimating the ELM model parameters we employed the well known Recursive Least Squares (RLS) [19].

\section{Simulation Results}

\section{A. Off-Line Comparison}

To evaluate the estimation performance of ELM parametrization, described by (12), two tests were carry out. The first test, presented in this section, is an "off-line" comparison between the ELM and previous models described in the literature (see Table I); the second test, described in the next section, is based on an "on-line" evaluation with the CarSim program, which provides a more realistic test environment.

For the off-line test, the training samples were artificially generated using the non-linear Magic Tyre Formula model, described by (5), in different types of roads: dry and wet asphalt, cobbles and snow, with parameters defined in Table II. To approximate the measuring uncertainty, the variable $\mu$ was corrupted with Gaussian noise, with zero mean and variance $0.06^{2}$. The performance indices used to evaluate the estimators were the absolute estimation errors in $\mu_{\max }$ and $\lambda_{\max }$ :

$$
\begin{aligned}
e_{\mu} & =\frac{\left|\mu_{\max }-\hat{\mu}_{\max }\right|}{\mu_{\max }} \\
e_{\lambda} & =\frac{\left|\lambda_{\max }-\hat{\lambda}_{\max }\right|}{\lambda_{\max }}
\end{aligned}
$$

The first step performed in the simulation was to assess the $\mu_{\max }$ and $\lambda_{\max }$ estimation performance offered by methods. During these simulations, it was verified that all the methods can estimate $\mu_{\max }$ with maximum errors inferior to $10 \%$. This result was expected, since the main motivation for introducing most of parametric models was to predict $\mu_{\max }$. For this reason the $\mu_{\max }$ estimation results will be omitted in this section. 

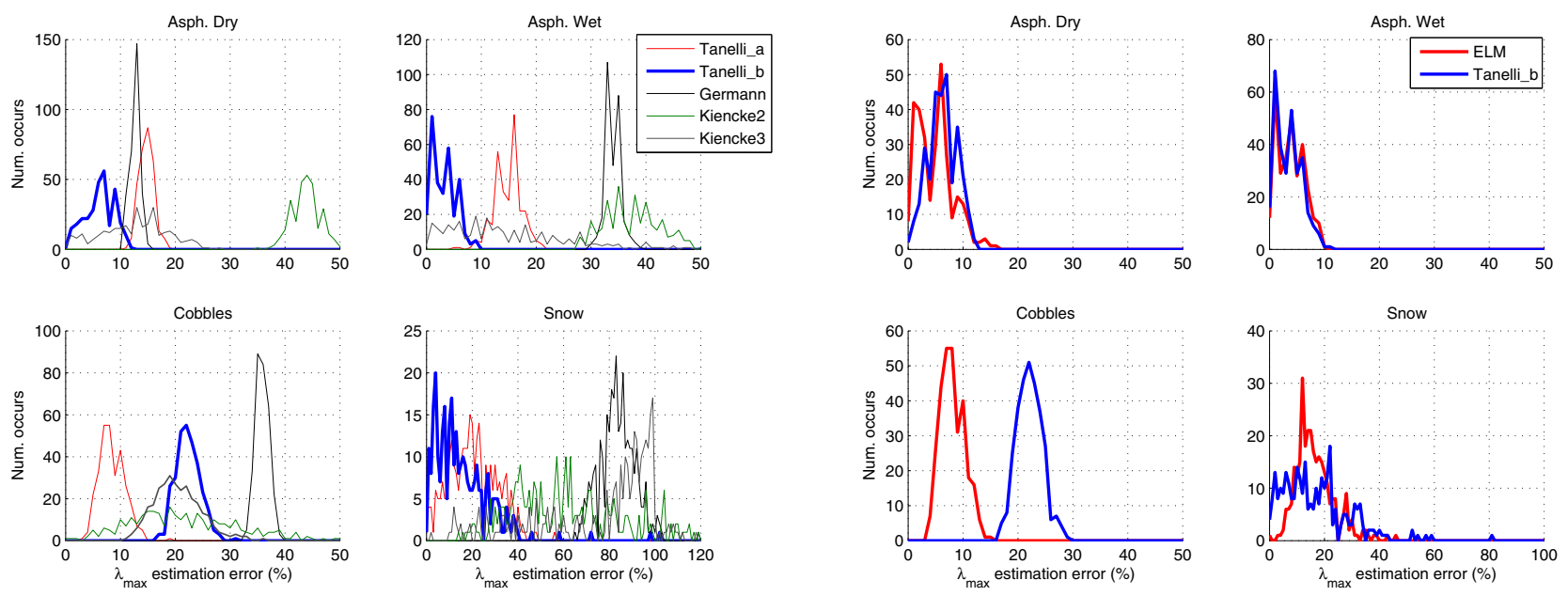

(a)

(b)

Figure 4. Histogram of the $\lambda_{\max }$ estimation error, in different adhesion conditions and using artificially generated samples by the MTF (see Table II). The parametrization under evaluation are described in Table I and, for each adhesion condition, 300 sets of samples with different noise realizations were generated.

However, as can be seen in Figure 4(a), the estimation methods shows higher errors in $\lambda_{\max }$ estimation. It is clear that, from all the parameterizations previously proposed in the literature and considered in this work, the Tanelli parameterizations are those that produce lower estimation errors, as a result of using non-linear models (Tanelli_a) or due to an increase in the number of parameters in the linear model (Tanelli_b). With the exception of cobbles roads, the Tanelli_b parametrization generates lowest $\lambda_{\max }$ estimation errors.

Figure 4(b) shows a comparison between the Tanelli_b and the method proposed in this article, the ELM. It can be seen that the ELM improves the $\lambda_{\max }$ estimation performance in cobbles, without penalizing the estimation performance in other types of roads, and using a model with less number of parameters than Tanelli_b.

\section{B. On-Line Simulation}

As a final evaluation test of the ELM model, a complete vehicle dynamics simulator was used, the CarSim [20], providing a more realistic testing environment. The simulation setting is based on series of braking maneuvers carried out on a variable grip road: the first part was run on dry asphalt and then progressing on wet asphalt. It was assumed that the measures (or estimates) of the instantaneous values of the tire slip and friction coefficient are available for the peak friction estimation.

The first time the vehicle brakes in a dry road (Figure 5a), there is no previous information about the friction model. Since in this test we are using the recursive least squares (RLS), the initial parameters estimates are crucial for the algorithm performance. In order to generate an initial guess $(\hat{\boldsymbol{\theta}}(0))$ for the model parameters we applied the traditional least squares to the first 20 samples having $\lambda<0.075$ (as suggested in [7]). For that reason, no estimation is produced during the first 0.1 seconds; after this initialization step, the RLS is activated and it can be seen good convergence rate to the true peak friction value $\left(\lambda_{\max }, \mu_{\max }\right)$, with final estimation errors inferior to $10 \%$.

A more challenging test is performed in the second part of the run, when the vehicle enter in the wet asphalt (Figure 5b). Since the vehicle has been previously running on dry asphalt, it is reasonable that the estimation algorithm uses the parameters obtained during the last braking maneuver (on dry) as the initial guess $(\hat{\boldsymbol{\theta}}(0))$ for the model. For that reason, the initial peak friction estimates (Figure $5 \mathrm{~d}$ ) are close to $\mu_{\max } \simeq 1$ and $\lambda_{\max } \simeq 0.12$, corresponding to the dry asphalt friction. However, when new samples are received by the estimator, we can see a decrease in the estimation error, converging, in $0.6 \mathrm{~s}$, to the true peak friction point of wet asphalt $\left(\mu_{\max } \simeq 0.5\right.$ and $\left.\lambda_{\max } \simeq 0.05\right)$, much inferior to the dry conditions. The simulation results presented in this section confirms the good performance offered by the ELM parameterization.

\section{CONCLUSions}

A new linear parametrization for the static representation of the friction coefficient was presented, targeting on-line estimation of the peak friction. This new model, based on a neural network and trained with the extreme learning machine methodology, needs only 4 parameters to describe the static friction curve $\mu($.$) , making it suitable for real-time$ implementation. Simulation results obtained with a complete vehicle dynamics simulator confirms a good performance of the proposed method.

As future work, we intent to experimentally validate the linear parametrization and apply the ELM to extract a linear identifiable model of the full magic tyre formula [3]. 

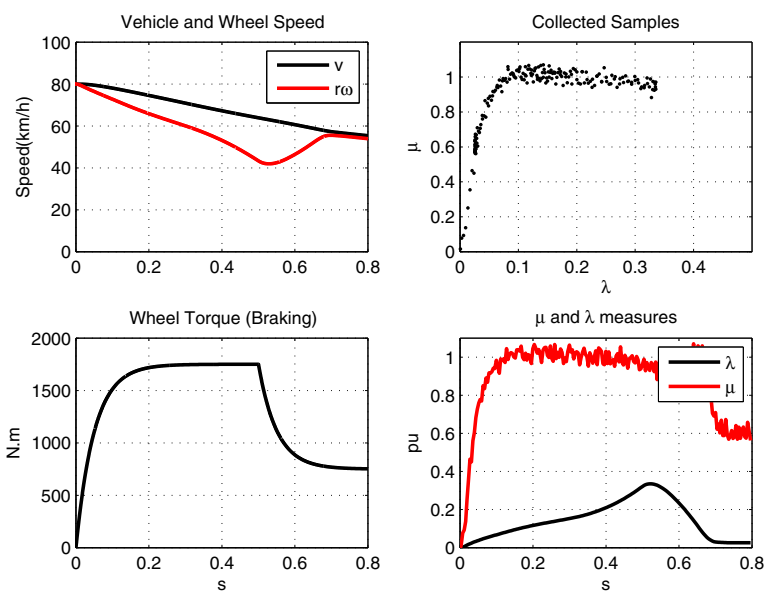

(a) Asph. Dry
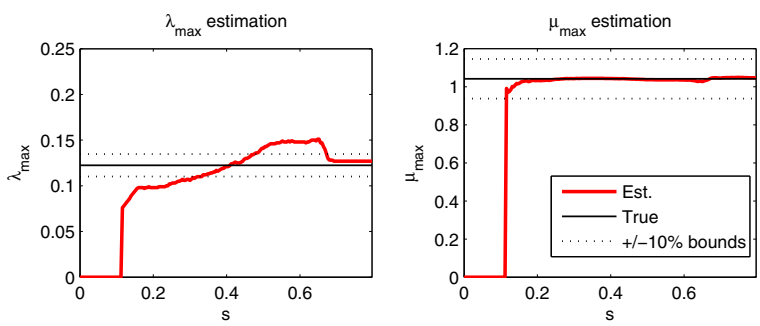

(c) ELM estimation (Asph. Dry)
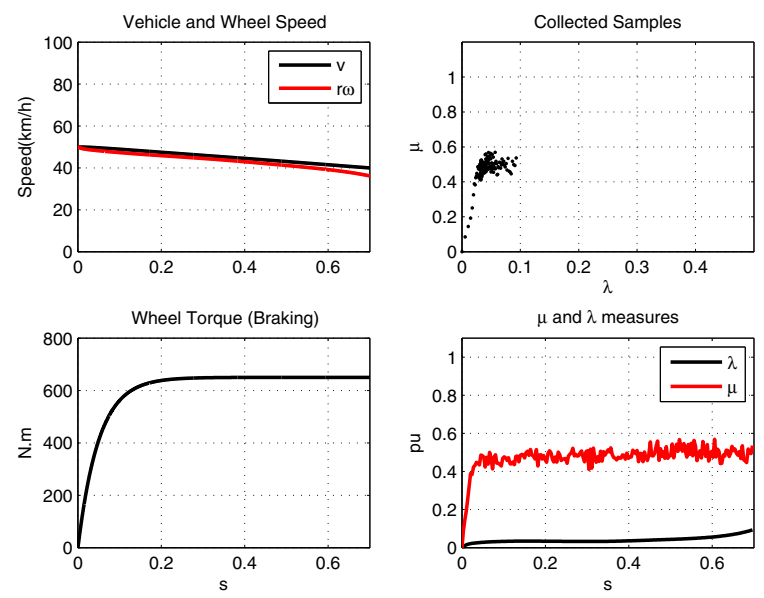

(b) Asph. Wet
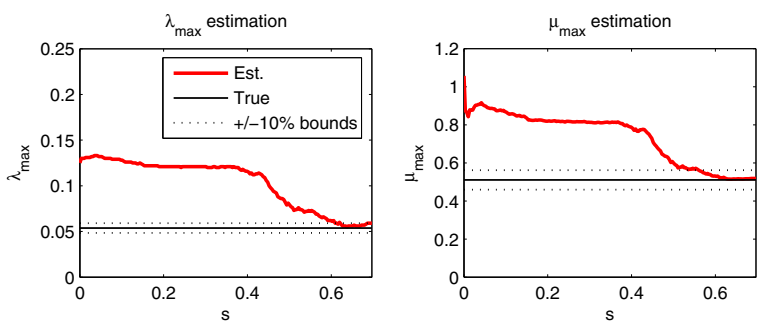

(d) ELM estimation (Asph. Wet)

Figure 5. Estimation of $\lambda_{\max }$ and $\mu_{\max }$, during braking maneuvers in dry asphalt (figures on the left side) and wet asphalt (figures on the right side).

\section{ACKNOWLEDGMENTS}

This work is partially funded by FCT - Science and Technology Foundation, through the project MIT-Pt/EDAMSMS/0030/2008.

\section{REFERENCES}

[1] A. T. van Zanten, "Evolution of electronic control systems for improving the vehicle dynamic behavior," in Proceedings of the International Symposium on Advanced Vehicle Control (AVEC), Hiroshima, Japan, 2002, pp. 7-15.

[2] M. Uchanski, "Road Friction Estimation for Automobiles Using Digital Signal Processing Methods," Ph.D. dissertation, University of California, Berkeley, 2001.

[3] H. B. Pacejka, Tyre and vehicle dynamics. Butterworth-Heinemann, 2002.

[4] U. Kiencke and L. Nielsen, Automotive Control Systems For Engine, Driveline, and Vehicle. Springer-Verlag, 2005.

[5] U. Kiencke, "Realtime estimation of adhesion characteristic between tyres and road," in Proc. of the IFAC 12th Triennial World Congress, vol. 1, 1993, pp. 15-22.

[6] S. Germann, M. Wurtenberger, and A. Daiss, "Monitoring of the friction coefficient between tyre and road surface," in Proceedings of the Third IEEE Conference on Control Applications, 1994.

[7] M. Tanelli, L. Piroddi, and S. M. Savaresi, "Real-time identification of tire-road friction conditions," Control Theory and Applications, IET, vol. 3, no. 7, pp. 891-906, 2009.

[8] S. Haykin, Neural networks: a comprehensive foundation. Prentice Hall, 1999.

[9] G.-B. Huang, Q.-Y. Zhu, and C.-K. Siew, "Extreme learning machine: Theory and applications," Neurocomputing, vol. 70, no. 1-3, pp. 489501, 2006.
[10] L. Nan-Ying, G.-B. Huang, P. Saratchandran, and N. Sundararajan, “A Fast and Accurate Online Sequential Learning Algorithm for Feedforward Networks," Neural Networks, IEEE Transactions on, vol. 17, no. 6, pp. 1411-1423, 2006.

[11] C. Canudas-de Wit, P. Tsiotras, E. Velenis, M. Basset, and G. Gissinger, "Dynamic friction models for road/tire longitudinal interaction," Vehicle System Dynamics, vol. 39, no. 3, pp. 189-226, 2003.

[12] L. Li and F.-Y. Wang, Advanced motion control and sensing for intelligent vehicles. Springer Verlag, 2007.

[13] F. Gustafsson, "Slip-based tire road friction estimation," Automatica, vol. 33, no. 6, pp. 1087-1099, 1997.

[14] K. Li, J. A. Misener, and K. Hedrick, "On-board road condition monitoring system using slip-based tyre-road friction estimation and wheel speed signal analysis," Proceedings of the Institution of Mechanical Engineers Part K-Journal of Multi-Body Dynamics, vol. 221, no. 1, pp. 129-146, 2007.

[15] E. Ono, K. Asano, M. Sugai, S. Ito, M. Yamamoto, M. Sawada, and Y. Yasui, "Estimation of automotive tire force characteristics using wheel velocity," Control Engineering Practice, vol. 11, no. 12, pp. 1361-1370, 2003.

[16] S. Muller, M. Uchanski, and K. Hedrick, "Estimation of the maximum tire-road friction coefficient," Journal of Dynamic Systems, Measurement, and Control, vol. 125, no. 4, pp. 607-617, 2003.

[17] C. R. Carlson and J. C. Gerdes, "Consistent nonlinear estimation of longitudinal tire stiffness and effective radius," Control Systems Technology, IEEE Transactions on, vol. 13, no. 6, pp. 1010-1020, 2005.

[18] Y. Hori, "Future vehicle driven by electricity and control - Research on four-wheel-motored UOT Electric March II," Industrial Electronics, IEEE Transactions on, vol. 51, no. 5, pp. 954-962, 2004.

[19] L. Ljung, System Identification: Theory for the User. Prentice Hall, 1999.

[20] “CarSim 8.0 User Manual,” Mechanical Simulation Corporation, 2009, http://www.carsim.com. 